\title{
Magnetite nanoparticles yield a significant bacteriostatic effect on microorganisms in relation to free radical lipid peroxidation
}

\author{
Belousov $A^{1}, Y_{\text {Y Voyda }}{ }^{2}$ and Belousova $E^{1}$ \\ ${ }^{1}$ Laboratory of Applied Nanotechnology of Belousova, Ukraine \\ ${ }^{2}$ Kharkov Medical Academy of Postgraduate Education, pr. Lenina, 31-v, fl. 32, Kharkov, 61072, Ukraine
}

\begin{abstract}
The influence of basic physical factors caused by magnetite nanoparticles (constant magnetic field and sorption) on microorganisms by examining the reactions of the intensity of free radical lipid peroxidation (FRLP) and bacteriostatic action was studied. It was well established that the magnetite nanoparticles caused unequal reaction in intensity of FRLP on different groups of microorganisms. It was determined that the most significant factor that influenced on the ultimate indicator of the intensity of luminescence on Candida albicans, Escherichia coli and Pseudomonas aeruginosa was constant magnetic field which induced by nanoparticles. On the contrary, sorption was the most significant factor on staphylococcus aureus. It was found that the rate of consumption of free radicals lipid reduced reliably on all microorganisms after their processing by magnetite nanoparticles. The results of microbiological studies of Escherichia coli, Klebsiella pneumoniae and Staphylococcus aureus showed that bacteriostatic effect was detected after exposure by magnetite nanoparticles. Visually, it was detected by decreasing the number of colonies on the nutritious medium in comparison with the control. It was revealed an interesting fact that physiological salt solution (saline $\mathrm{NaCl}$ ), which had previously been processed by magnetite nanoparticles also significantly had a marked bacteriostatic effect on the studied microorganisms. This effect could be explained by mechanism of change the polarization structure water of microorganisms by magnetite nanoparticles. It was discovered that degree of expression of bacteriostatic action which induced by magnetite nanoparticles had correlation with marks of reactions intensity of FRLP. Maximum bacteriostatic effect on staphylococcus aureus was expressed in second variant application of magnetite nanoparticles where mechanism of sorption was more significant than action of the magnetic field. On the contrary, maximum bacteriostatic effect on Escherichia coli and Klebsiella pneumoniae was revealed in third variant, where time exposition of contact with microorganisms nanoparticles and, consequently, action of a constant magnetic field was determinative.
\end{abstract}

\section{Introductions}

Today the world's attention is locked on the problem of bacterial resistant to antibiotics - one of the most serious threats to humanity. Chief Medical Officer, Dame Sally Davies, warned that if this problem didn't solved than in 20 years' time even minor surgery might lead to death through untreatable infection. In Britain, in spite of greatly reducing hospital infections from bugs such as MRSA (methicillin resistant staphylococcus aureus) and clostridium difficile to the levels of 10 years ago - falling by 80 per cent - they were being replaced by opportunistic bacteria such as Escherichia coli and Klebsiella pneumoniae, which became one of the most frequent agents of nosocomial infections. About 5.000 patients a year in the UK died from bloodstream infections, half of them caused by drug-resistant organisms. Antimicrobial resistance is a ticking time-bomb not only for the UK but also for the whole world. Many experts believe that in the future all antibiotics would become useless and humanity had to refuse of taking them [1]. Even now, the numbers of new antibiotics have been reduced dramatically. Antibiotic resistance leads to cases when people get sick for a long time, and increases vital infections. Resistant microorganisms also lead to increasing spends of medical aid because of a prolonged stay in the clinics and this situation requires treatment that is more intensive. The development of new antibacterial medicines based on nanoparticles is one of the real ways to solve this problem. The first scientific publications on the investigation influence of magnetite nanoparticles on microorganisms appeared in 1998. Reliable information about the bacteriostatic effect of magnetite nanoparticles to certain pathogens was represented. Also, it was discovered that magnetite nanoparticles significantly increase the sensitivity of pathogens to antibacterial medications [2]. Action of nanoparticles and nanocapsules on microorganisms was tested before. The bacteriostatic and bactericidal effects of nanoparticles were confirmed [3-5]. Furthermore, an example of studying human erythrocytes showed that the magnetite nanoparticles altered reliably the polarizable structure of the water sector of cell microenvironment and as a result their permeability [6].

Thus, analysis of above-mentioned dates could give us the opportunity of highly optimistic talking about the future development of a new direction - elaboration of antibacterial medications based on magnetite nanoparticles. The mechanism of their action will be associated with changes of physical factors of the cell existence. But today mechanisms of physical influence of magnetite nanoparticles on

*Correspondence to: PProf. Andrey Belousov, Head Laboratory of Applied Nanotechnology, Pr. Lenina, 31-v, fl. 32 Kharkov, 61072, Ukraine, Tel: 38050 915-18-89; E-mail: an.belousov2012@yandex.ua; andrey.belousov@eng-invest.at

Key words: magnetite nanoparticles, microorganisms, free radicals peroxidation lipids, polarization structure, bacteriostatic effect

Received: August 28, 2016; Accepted: September 20, 2016; Published: September 23, 2016 
microorganisms are still poorly understood, and the main purpose of our investigations was to find out them.

The main goal was to investigate the influence of basic physical factors of magnetite nanoparticles on different groups of microorganisms.

In order to perform this goal next tasks were set:

- to investigate the effect of magnetite nanoparticle on the intensity of free radical peroxidation lipids (FRPL) in various bacterial cultures;

- to explore direct bacteriostatic action of magnetite nanoparticle on microorganisms;

- to study bacteriostatic effect of physiological salt solution (saline $\mathrm{NaCl}$ ) that had previously been processed by magnetite nanoparticles;

- to establish a causal link between research results and main physical factors of magnetite nanoparticles.

\section{Materials and methods}

\section{Materials}

Colloidal solution of magnet-controlled sorbent (MCS-B). The basis of MCS-B is magnetite nanoparticles $\left(\mathrm{Fe}_{3} \mathrm{O}_{4}\right)$. The size of nanoparticles is from 6 to $12 \mathrm{~nm}$; the total sorption surface of NPs is from 800 to 1200 $\mathrm{m}^{2} / \mathrm{g}$; magnetization of saturation $\mathrm{I}=2.15 \mathrm{kA} / \mathrm{m}$; volume concentration $\mathrm{q}=0.00448$; viscosity $\eta=1.0112 \mathrm{cSt} ; \zeta$ - potential=-19 mV; saline $\mathrm{NaCl}$ that had previously been processed by magnetite nanoparticles. Magnetite nanoparticles have been produced in Laboratory of Applied Nanotechnology of Belousova.

\section{Object of research}

Candida albicans ATCC 885/653, Escherichia coli ATCC 25922, Pseudomonas aeruginosa ATCC 9027, Staphylococcus aureus ATCC 25923, Klebsiella pneumonia ATCC 700603.

\section{Research methods}

The intensity of free radical peroxidation lipids (FRPL) was measured by means method of hemiluminescence for installing quantmetric equipment National Science Center "Kharkov Institute of Physics and Technology" with multi-channel photomultiplier PMT140 [7]. The studied object was placed in thermostatic cuvette at $37^{\circ} \mathrm{C}$, which was located at the time measurement of the photomultiplier (PMT) cathode end. The intrinsic noises of the PMT were in the range of 30-70 pulses/sec. The various kinds of hemiluminescencent reaction were used, which allowed to discover the nature of the various content oxygen products of FRPL. Chemiluminescent determination of superoxide anion radical was carried out in the luminol + bacterial medium. In a thermostatic cuvette chemiluminometer placed $1.5 \mathrm{ml}$ $0.075 \%$ luminol $+0.25 \mathrm{ml}$ of bacterial medium $+0.25 \mathrm{ml}$ of a $0.1 \%$ solution of magnetite nanoparticles. Induction was carried out by

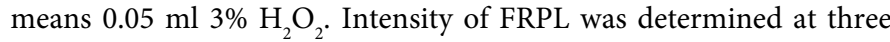
stages: I stage - I max determined, II stage - I final, III stage - the sum of light $\sum$.

\section{Preparation of bacterial cultures}

After $24 \mathrm{~h}$ bacterial cultures growth on Mueller-Hinton agar was made microbial suspension in saline $\mathrm{NaCl}$ with scale $\mathrm{McF}$ arland appropriate 0.5 . Then the initial concentration of working culture was titrated to concentration corresponding to $10^{4}$. Sowing $S$. aureus was carried out on Mueller-Hinton medium; E.coli and K. pneumoniae - on Endo medium.

\section{Bacteriostatic effect has been studied in the following variants}

variant 1 - control; variant 2 - the study of bacteriostatic action of magnetite nanoparticles (MCS-B) after their short-term contact with microorganisms; variant 3 - after 24 hours contact of microorganisms with MCS-B; variant 4 - after 24 hours contact of microorganisms with saline of $\mathrm{NaCl}$, that had previously been processed by MCS-B. Estimate was carried out in comparison with the control.

Statistically processing the obtained results was performed by parametrical method of variation statistics by Student criterion.

\section{Results and discussion}

The results of the research intensity of FRPL in various bacterial mediums are presented in Table 1.

The date in Table 1 showed that intensity of the first flash of luminescence after the introduction of $\mathrm{H}_{2} \mathrm{O}_{2}$ (I max) for all studied microorganisms after processed by magnetite nanoparticles was $(\mathrm{p}<0.001)$ reduced reliably by $2-2.5$ times in comparison with the control. This happened due to a decrease in the total amount of lipid peroxidation products as a result of adsorption by magnetite nanoparticles [8].

However, it was not determined reliable differences at stage II of the I final index in compared with control on microorganisms such as Candida albicans, Escherichia coli and Pseudomonas aeruginosa $(\mathrm{p}>0.05)$. On the contrary, I final index differed reliably $(\mathrm{p}<0.001)$ from the control on Staphylococcus aureus. This fact indicated that the

Table 1. Indicators of the intensity of free radical peroxidation in variants bacterial cultures at stages of the study $(M \pm m ; n=10)$.

\begin{tabular}{|c|c|c|c|}
\hline Type of object & $\begin{array}{l}\text { I max, } \\
\text { imp/sec }\end{array}$ & $\begin{array}{l}\text { I final, } \\
\text { imp/sec }\end{array}$ & $\begin{array}{c}\text { Sum of light } \\
\sum\end{array}$ \\
\hline Candida albicans & $16385 \pm 210$ & $720 \pm 98$ & $2520 * 10^{2} \pm 210$ \\
\hline Candida albicans + magnetite nanoparticles & $6646 \pm 178^{*}$ & $700 \pm 76^{* * *}$ & $1745 \times 10^{2} \pm 100^{*}$ \\
\hline Escherichia coli & $2086 \pm 99$ & $583 \pm 90$ & $1452 \times 10^{2} \pm 97$ \\
\hline Escherichia coli + magnetite nanoparticles & $827 \pm 50^{*}$ & $432 \pm 88 * * *$ & $1063 \times 10^{2} \pm 102^{* *}$ \\
\hline Staphylococcus aureus & $7384 \pm 122$ & $1179 \pm 70$ & $3748 \times 10^{2} \pm 171$ \\
\hline Staphylococcus aureus + magnetite nanoparticles & $4471 \pm 121 *$ & $673 \pm 70^{*}$ & $2625 \times 10^{2} \pm 154^{*}$ \\
\hline Pseudomonas aeruginosa & $6245 \pm 102$ & $820 \pm 65$ & $3215 \times 10^{2} \pm 200$ \\
\hline Pseudomonas aeruginosa + magnetite nanoparticles & $2648 \pm 99^{*}$ & $778 \pm 55^{* * *}$ & $2734 \times 10^{2} \pm 132 * *$ \\
\hline
\end{tabular}

Note: $*$ - $\mathrm{p}<0.001 ; * *-\mathrm{p}<0.05 ; * * *-\mathrm{p}>0.05$

I max. - intensity first flash luminescence (imp/sec) after the introduction of $\mathrm{H}_{2} \mathrm{O}_{2}$ in the first minute. The maximum value of the first flash gives an indication of the total number of peroxidation products in the substrate.

I final - ultimate intensity of luminescence (imp/sec) after 4 minutes. This index corresponds to the amount of the remaining unreacted peroxidation products or antioxidants. $\sum$ - sum of light in 4 minutes measurement. This index indicates to rate of consumption of the lipid nature free radicals in consequence their interaction with antioxidants. 
sorption activity of magnetite nanoparticles in the bacterial culture of staphylococcus aureus was more important factor in the physical action than the effect of a constant magnetic field.

Stage III study (determine $\Sigma$ - sum light in 4 minutes) demonstrated that after processed of bacterial medium by magnetite nanoparticles the consumption speed of free radical lipid was reduced reliably on all microorganisms. This fact was connected with sorption effect that supplemented by the action of the magnetic field of the nanoparticles. The constant magnetic field that induced (power of 300-400 kA/m) deprotonated (transfer in active status) enzymes of antiradical defend. As a result the synthesis of lipid peroxides and secondary radical reactions were blocked, inhibited lipid peroxidation [9].

Indicators the decline in the intensity of free radical peroxidation in bacterial cultures were supplemented with data from research of bacteriostatic action of magnetite nanoparticles (Figure 1).

Figure 1 could demonstrate that bacteriostatic effect on bacterial cultures such as E. coli and K. pneumonia was determined after 24 hours exposition of magnetite nanoparticles with microorganisms. As a result there was significant decline in the number of colonies on the nutritious medium.

Maximum bacteriostatic action on $S$. aureus was found in the variant of short-term contact with nanoparticles of microorganisms (variant 2).

If mechanism of bacteriostatic effect in the second and third variants could be explained from position of the sorption activity of nanoparticles, in the fourth variant, where microorganisms contacted only with saline $\mathrm{NaCl}$, which had previously been processed by nanoparticles, the sorption action was excluded. The mechanism for this effect was explained by increased mobility of the hydrogen protons and changing their spatial orientation after processed by magnetite nanoparticles. As a result, the polarizable structure of the cell water was modified and changed its permeability [10]. After analyzing all received data of research, we made a conclusion that degree of expression of bacteriostatic action had correlation with marks of reactions intensity of FRLP.

So, maximum bacteriostatic effect on Staphylococcus aureus was expressed in second variant application of magnetite nanoparticles

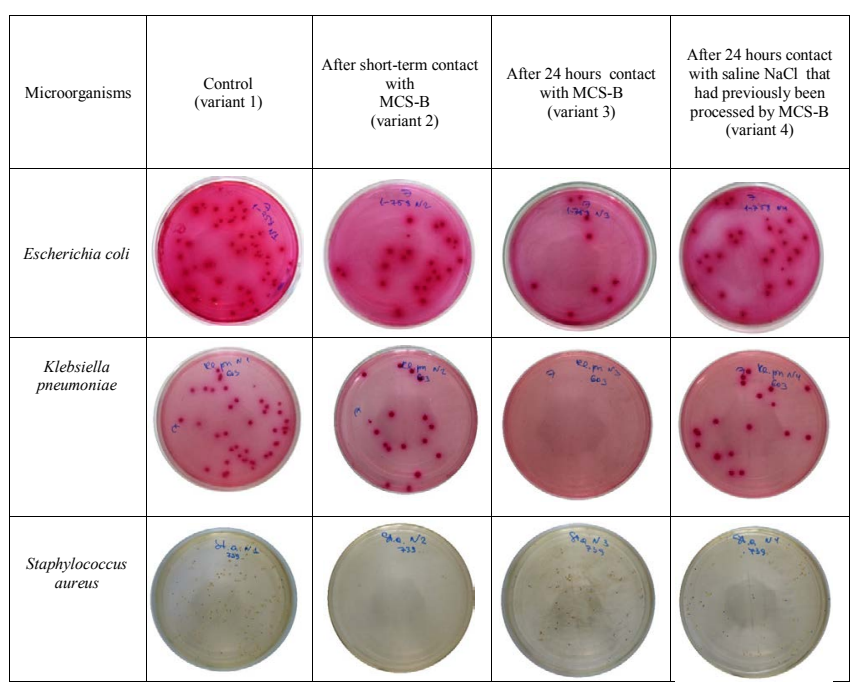

Figure 1. Study bacteriostatic action of magnetite nanoparticles (MCS-B) and saline $\mathrm{NaCl}$ that was processed by nanoparticles on different groups of microorganisms. where mechanism of sorption was more significant than action of the magnetic field. On the contrary, maximum bacteriostatic effect on Escherichia coli and Klebsiella pneumoniae was revealed in third variant, where time exposition of contact with microorganisms nanoparticles and, consequently, action of a constant magnetic field was determinative. Received data confirmed the strength of influence of magnetic field caused by the nanoparticles on the structure of water by the fact of bacteriostatic action of saline $\mathrm{NaCl}$, which had previously been processed by magnetite nanoparticles.

Thus, it was found effects of the basic physical factors of magnetite nanoparticles (constant magnetic field and sorption) on different groups of microorganisms and fact that both factors had an impact on microorganisms, but determined influence of different factors would depend reliably on the individual properties of studied microorganisms.

\section{Conclusions}

1. It was expanded the range of studying the mechanisms of the influence of physical factors of magnetite nanoparticles on microorganisms by examining the reactions intensity FRPL and bacteriostatic action.

2. It was established reliably that magnetite nanoparticles on different groups of microorganisms caused unequal intensity FRPL reaction.

It was determined that more significant factor that influenced on the ultimate indicator of the intensity of luminescence on Candida albicans, Escherichia coli and Pseudomonas aeruginosa was constant magnetic field which induced by nanoparticles. On the contrary, sorption was the most significant factor on staphylococcus aureus.

3. It was found that the rate of consumption of free radicals lipid reduced reliably in all microorganisms after their processing by magnetite nanoparticles.

The results of microbiological studies of Escherichia coli, Klebsiella pneumoniae and Staphylococcus aureus showed that bacteriostatic effect was detected after exposure by magnetite nanoparticles. Visually, it was detected by decreasing the number of colonies on the nutritious medium in comparison with the control.

4. It was revealed an interesting fact that saline $\mathrm{NaCl}$, which had previously been processed by magnetite nanoparticles also significantly, had a marked bacteriostatic effect on the studied microorganisms. This effect could be explained by mechanism of change the polarizable structure water of microorganisms by magnetite nanoparticles.

It was discovered that degree of expression of bacteriostatic action which induced by magnetite nanoparticles had correlation with marks of reactions intensity of FRLP. Maximum bacteriostatic effect on staphylococcus aureus was expressed in second variant application of magnetite nanoparticles where mechanism of sorption was more significant than action of the magnetic field. On the contrary, maximum bacteriostatic effect on Escherichia coli and Klebsiella pneumoniae was revealed in third variant, where time exposition of contact with microorganisms nanoparticles and, consequently, action of a constant magnetic field was determinative.

\section{References}

1. http://www.independent.co.uk/news/science/chief-medical-officer-dame-sally-daviesresistance-to-antibiotics-risks-health-catastrophe-to-rank-with-terrorism-and-climatechange-8528442.html 
2. Belousov AN, Osolodchenko TP, Belousova Ye Yu (1998) Effects of magtietic fluid preparations on microorganisms // Eighth International Conference on magnetic fluids. - Timisoara, Romana. 486-487.

3. Lopes LQ, Santos CG, de Almeida Vaucher R, Gende L, Raffin RP, et al. (2016) Nanocapsules with glycerol monolaurate: Effects on Candida albicans biofilms. Microb Pathog 97: 183-188. [Crossref]

4. Kalita S, Devi B, Kandimalla R, Sharma KK, Sharma A, et al. (2015) Chloramphenicol encapsulated in poly-î́ $\mu$-caprolactone-pluronic composite: nanoparticles for treatment of MRSA-infected burn wounds. Int J Nanomedicine 10: 2971-2984. [Crossref]

5. Mata R1, Reddy Nakkala J1, Rani Sadras S2 (2015) Catalytic and biological activities of green silver nanoparticles synthesized from Plumeria alba (frangipani) flower extract. Mater Sci Eng C Mater Biol Appl 51: 216-225. [Crossref]

6. Belousov A. The Role of Magnetite Nanoparticles (ICNB) in Discovery New Factor,
Which Influence on Permeability of Erythrocytes and Eryptosis. OALibJ 8: 1-4.

7. Gerasimov AM, Kornev EN, Amelin DSh (1978) Modeling the relationship of peroxide generating and NADPH - dependent processes. Oxidative enzymes of animal cells and the regulation of their activity. Symp of Russia Gorkiy: 23-24.

8. Belousov A (2001) Effect of extracorporeal hemocorrection with using magnetcontrolled sorbent on the intensity of lipid peroxidation. Experimental and Clinical Medicine. Kharkov State Medical University 113-115.

9. Belousov A (2004) The extracorporal hemocorrection with application of magnetcontrolled sorbent in intensive therapy of syndrome intoxication in the patients with a pathology hepatopancreatoduodenum zones: The abstract dissertation of doctor medicine: 14.01.30. / The Dnepropetrovsk state of medical academy.

10. Belousov A (2014) Inhibition of Eryptosis by Means of Magnetite Nanoparticles (MCS-B). International Journal of Advance in Medical Science (AMS) 2: 19-23.

Copyright: $(02016$ Belousov A. This is an open-access article distributed under the terms of the Creative Commons Attribution License, which permits unrestricted use, distribution, and reproduction in any medium, provided the original author and source are credited. 